\title{
Documentos
}

\section{NORMAS DE PROFILAXIS ANTIBIÓTICA EN PROCEDIMIENTOS OBSTÉIRICOS}

\author{
Hugo Salinas $P^{1,2}$, Jessica Preisler $R .^{1}$, Cristina Aleuanlli A. ${ }^{1, a}$, Rodolfo Ide V. ${ }^{1}$, Marcia \\ Erazo B. ${ }^{2, b}$, Sergio Carmona G. ${ }^{3}$, Daniel Erlij O. ${ }^{c}$, Paulina Sepúlveda $R^{c}$ \\ ${ }^{1}$ Departamento de Obstetricia y Ginecología, Hospital Clínico de la Universidad de Chile. ²Programa de Doctorado en \\ Salud Pública, Escuela de Salud Pública de la Universidad de Chile. ${ }^{3}$ Departamento de Obstetricia y Ginecología, \\ Hospital Clínico San Borja Arriarán.
}

aMatrona. ${ }^{b}$ Enfermera. 'Internos de Medicina, Universidad de Santiago.

\section{RESUMEN}

La terapia antibiótica profiláctica en los actos quirúrgicos es una práctica común y aceptada por la comunidad médica, por que disminuye la probabilidad de infección quirúrgica y las complicaciones implican. A pesar de la frecuencia de uso y las normas hospitalarias que lo regulan, es posible observar la falta de seguimiento de éstas por parte del personal médico, quienes ateniéndose a conocimientos personales, al seguimiento de normas de otro origen o simplemente al desconocimiento de ellas, utilizan criterios y esquemas distintos a los recomendados, lo que aumenta el riesgo de utilizar terapias inapropiadas, la resistencia bacteriana a los antibióticos y los costos. El siguiente estudio compara el uso de antibióticos en procedimientos y cirugías obstétricas establecidas en el Servicio de Obstetricia del Hospital Clínico de la Universidad de Chile con las normas propuestas por el $\mathrm{NIH}$, para así proponer una norma sobre el uso racional de antibióticos profilácticos en cirugía obstétrica.

\section{PALABRAS CLAVES: Profilaxis antibiótica, cirugía obstétrica}

\section{SUMMARY}

The use of the prophylactic antibiotic therapy in the surgical act is a common and accepted practice among the medical community, representing a tool that lowers the probability of infection in the surgical site, at a distance and the complications it implies. In spite of the frequency of its use and the establishment of hospital norms that regulate it, it is possible to observe the lack of follow-up of these by the medical personnel, who usually rely on personal knowledge, norms of another origin or simply on the ignorance of the regulations, and use criteria and schemes different from the recommended ones, which increases the risk of bacterial resistance, using unsuitable therapies, and raises the costs. The following study compares the use of antibiotics in surgical procedures and obstetrical surgeries in the obstetrics service of the Hospital Clínico de la Universidad de Chile with the norms of this center and international ones by the $\mathrm{NIH}$, thus to propose a norm on rational use of prophylactic antibiotics in obstetrical surgery.

KEY WORDS: Antibiotic prophylaxis, obstetrical surgery 


\section{INTRODUCCIÓN}

El uso de antibióticos profilácticos en procedimientos quirúrgicos se ha utilizado desde los años 50 con el objetivo de prevenir infecciones postoperatorias (1). Además de su efecto en la reducción de infección de la herida operatoria, se describe que disminuirían otras complicaciones como neumonía, endocarditis e infección urinaria (2).

En obstetricia, el uso de antibióticos profilácticos tendría un rol en reducir la incidencia de infección en pacientes con abortos espontáneos o provocados, que requieren de legrado uterino, y en pacientes sometidas a cesárea $(3,4)$ no estando recomendada en parto vaginal no complicado con o sin episiotomía ni en aborto espontáneo que no requiere maniobras instrumentales.

En el Hospital Clínico de la Universidad de Chile (HCUCH) (5), la profilaxis antibiótica en procedimientos obstétricos considera el uso en cesárea (con rotura prematura de membranas mayor de 12 horas, con trabajo de parto prolongado, 5 o más tactos vaginales previo a la cirugía) y en aborto provocado.

Si bien existen en la literatura numerosos estudios sobre profilaxis antibiótica en cirugía obstétrica y ginecológica, en nuestro medio no existe aún consenso sobre cual es la droga de elección para ser utilizada en cada procedimiento quirúrgico.

En $\mathrm{HCUCH}$, la recomendación en cesárea es la utilización de cefazolina 1 gramo ev después de ligar el cordón umbilical y en caso de alergia metronidazol $500 \mathrm{mg}$ ev en similar momento. En aborto provocado la indicación contempla penicilina G 1 millón de unidades ev más cloramfenicol 1 gramo ev cada 8 horas más gentamicina $1 \mathrm{mg} / \mathrm{Kg} /$ dosis cada 8 horas ev por 3 a 5 días (5).

No cabe ninguna duda que el concepto de profilaxis antibiótica es ampliamente aceptado, no así necesariamente las drogas utilizadas para dichos fines, ni menos aún, comprendiendo los beneficios de la utilización de esta estrategia, que efectivamente esté siendo correctamente utilizada.

Los objetivos del presente estudio son actualizar las indicaciones internacionales del uso profiláctico de antibióticos en cirugía obstétrica para su recomendación, analizar el tipo de profilaxis empleada en el $\mathrm{HCUCH}$ de acuerdo al Manual de Control de Infecciones Intrahospitalarias (5) y proponer una norma sobre el uso racional de antibióticos profilácticos en cirugía obstétrica.

\section{MATERIAL Y MÉTODO}

Se realizó una búsqueda en Internet en los sitios mdconsult, pubmed y ovid, bajo los términos "antibiotic prophylaxis in surgery", "antibiotics in obstetrics" y "antibiotics in gynecological surgery", encontrándose 2000 referencias. Se seleccionaron de estas referencias, 6 trabajos del año 2000 en adelante que cumplían los requisitos para ser consideradas revisiones sistemáticas del tema, con el objeto de consensuar esquemas de uso de antibióticos profilácticos en obstetricia.

Estos esquemas antibióticos recolectados en la literatura, se resumen en la propuesta del National Institut of Health $(\mathrm{NIH})$, de acuerdo al esquema que se muestra en la Tabla I y que sirvió de base para nuestro estudio.

Complementariamente se realizó un muestreo aleatorio representativo de la actividad del Servicio (7), analizando en forma retrospectiva 406 fichas clínicas de pacientes sometidas a intervenciones quirúrgicas obstétricas en el Servicio de Obstetricia del $\mathrm{HCUCH}$, durante el segundo semestre de 2004 y el primer semestre de 2005, tabulándose si hubo o no profilaxis antibiótica, y de haber sido utilizada, la droga o drogas empleadas, vías de administración y los plazos de administración.

\section{RESULTADOS}

Según las normas del National Institut of Health (NIH), la profilaxis antibiótica no está recomendada en parto vaginal no complicado con o sin episiotomía ni en aborto espontáneo, que no requieren maniobras instrumentales. Por el contrario, la profilaxis antibiótica se recomienda en cesárea y legrado uterino (Tabla I).

Durante el período de estudio y según el criterio empleado por $\mathrm{NIH}$, se analizaron 406 fichas clínicas, correspondiendo a 170 cesáreas, 123 partos vaginales eutócicos, 47 partos vaginales operatorios y 66 vaciamientos uterinos.

Del total de cesáreas, el 51,17\% fueron electivas; de éstas $98,8 \%$ recibieron profilaxis antibiótica, en comparación al $93 \%$ de las pacientes sometidas a una cesárea de urgencia. En ambos casos, el esquema más utilizado fue cefazolina 2 gramos $(83,8 \%$ y $72,7 \%$, en cesárea electiva y de urgencia, respectivamente).

Al comparar partos con y sin episiotomía, se evidenció uso de antibiótico profiláctico en 51 y $45 \%$ de las pacientes, respectivamente; similar a lo ocurrido en cesáreas, el esquema profiláctico más usado fue cefazolina 2 gramos. 
Tabla I

ESQUEMAS DE PROFILAXIS ANTIBIÓTICA EN PROCEDIMIENTOS OBSTÉTRICOS, SEGÚN NIH (6)

\begin{tabular}{|c|c|c|c|}
\hline Procedimiento & Esquema de elección & Duración & Alternativas \\
\hline Cesárea & $\begin{array}{l}\text { Cefazolina } 2 \mathrm{~g} \text { inmediata- } \\
\text { mente después de ligar el } \\
\text { cordón umbilical }\end{array}$ & Monodosis & $\begin{array}{l}\text { Alergia a } \beta \text {-lactámicos: } \\
\text { Clindamicina } 600 \mathrm{mg}+ \\
\text { Gentamicina } 1,5 \mathrm{mg} / \mathrm{kg} \\
\text { dosis única }\end{array}$ \\
\hline $\begin{array}{l}\text { Legrado para evacuación } \\
\text { de aborto espontáneo } \\
\text { primer trimestre }\end{array}$ & $\begin{array}{l}\text { Doxiciclina } 200 \mathrm{mg} \text { vo } 2 \text { horas } \\
\text { previas al procedimiento y } 200 \mathrm{mg} \\
\text { a las } 12 \text { horas de efectuado }\end{array}$ & 12 horas & $\begin{array}{l}\text { Alergia a } \beta \text {-lactámicos: } \\
\text { Metronidazol } 500 \text { mg vo } \\
2 \text { horas previas al procedimiento }\end{array}$ \\
\hline $\begin{array}{l}\text { Legrado para evacuación } \\
\text { de aborto espontáneo } \\
\text { segundo y tercer trimestre }\end{array}$ & $\begin{array}{l}\text { Cefazolina } 1 \mathrm{~g} \text { preinducción, } \\
\text { luego } 1 \mathrm{~g} \mathrm{c} / 8 \text { horas por } 24 \text { horas }\end{array}$ & 24 horas & $\begin{array}{l}\text { Alergia a } \beta \text {-lactámicos: } \\
\text { Clindamicina } 600 \mathrm{mg}+ \\
\text { Gentamicina } 1,5 \mathrm{mg} / \mathrm{kg} \text { preinción, } \\
\text { luego Clindamicina } 600 \mathrm{mg} \mathrm{c} 8 \mathrm{hs} \\
\text { hasta completar } 24 \text { horas + } \\
\text { Gentamicina } 3 \mathrm{mg} / \mathrm{kg} \text { dosis única }\end{array}$ \\
\hline
\end{tabular}

En el caso de parto distócico vaginal, $60 \%$ de las pacientes recibió terapia profiláctica con el mismo esquema antibiótico preferido en las cirugías antes descritas (cefazolina 2 gramos en $60,7 \%$ de los casos).

Finalmente, en $65 \%$ de los vaciamientos uterinos recibieron profilaxis antibiótica, sin embargo, el esquema utilizado en el vaciamiento de I y II trimestre fue distinto: en el primero se utilizó mayoritariamente cefazolina 1 gramo v/s 2 gramos en el segundo (Tabla II).

\section{DISCUSIÓN}

El uso de antibióticos profilácticos en $\mathrm{HCUCH}$, coincide en su mayoría con las recomendaciones del NIH y no así con las Normas de nuestro Hospital.
Una revisión sistemática sobre profilaxis antibiótica en cesáreas, comparó la eficacia de distintos esquemas antibióticos en la reducción de la incidencia de endometritis puerperal. Al comparar ampicilina $\mathrm{v} / \mathrm{s}$ cefalosporinas de primera, segunda y tercera generación, y además cefalosporinas de distintas generaciones entre si, se observó que no hubo diferencias estadísticamente significativas en la disminución de endometritis. Pero se sugiere usar cefalosporinas de primera generación o ampicilina, ya que no se justifica emplear antibióticos de mayor espectro de acción (8).

Por otro lado, se evaluó el efecto del uso de profilaxis antibiótica en las complicaciones infecciosas de pacientes sometidas a cesáreas electivas y de urgencia. Se observó una reducción significativa de la incidencia de fiebre postparto, endometritis, infección de herida operatoria e in-

Tabla II

PROFILAXIS ANTIBIÓTICA EN EL SERVICIO DE OBSTETRICIA DEL HOSPITAL CLÍNICO DE LA UNIVERSIDAD De CHILE (5)

\begin{tabular}{|c|c|c|c|c|c|c|c|c|c|c|c|}
\hline \multirow[t]{2}{*}{ Intervención } & \multicolumn{2}{|c|}{$\begin{array}{c}\text { Profilaxis } \\
\text { Sí }\end{array}$} & \multicolumn{2}{|c|}{$\begin{array}{c}\text { Profilaxis } \\
\text { No }\end{array}$} & \multicolumn{2}{|c|}{$\begin{array}{c}\text { ATB } \\
\text { Cefazolina } 1 \mathrm{~g}\end{array}$} & \multicolumn{2}{|c|}{$\begin{array}{c}\text { ATB } \\
\text { Cefazolina } 2 \mathrm{~g}\end{array}$} & \multicolumn{2}{|c|}{$\begin{array}{l}\text { ATB } \\
\text { otro }\end{array}$} & \multirow{2}{*}{$\begin{array}{c}\text { Total inter- } \\
\text { vención } \\
n\end{array}$} \\
\hline & $n$ & $\%$ & $n$ & $\%$ & $n$ & (\%) & $n$ & $(\%)$ & $n$ & (\%) & \\
\hline Cesárea electiva & 86 & 98,8 & 1 & 1,2 & 7 & $(8,1)$ & 72 & $(83,8)$ & 7 & $(8,1)$ & 87 \\
\hline Cesárea urgencia & 77 & 93 & 6 & 7 & 13 & $(16,9)$ & 56 & $(72,7)$ & 8 & $(10,4)$ & 83 \\
\hline Parto sin episiotomía & 18 & 45 & 22 & 55 & 8 & $(44,4)$ & 8 & $(44,4)$ & 2 & $(11,2)$ & 40 \\
\hline Parto con episiotomía & 87 & 51,2 & 83 & 48,8 & 27 & (31) & 57 & $(65,5)$ & 3 & $(3,5)$ & 170 \\
\hline Fórceps & 28 & 60 & 19 & 40 & 10 & $(35,7)$ & 17 & $(60,7)$ & 1 & $(3,6)$ & 47 \\
\hline Vaciamiento I trimestre & 37 & 63,8 & 21 & 36,2 & 23 & $(62,2)$ & 11 & $(29,7)$ & 3 & $(8,1)$ & 58 \\
\hline Vaciamiento II trimestre & 6 & 75 & 2 & 25 & 1 & $(16,7)$ & 4 & $(66,6)$ & 1 & $(16,7)$ & 8 \\
\hline
\end{tabular}

ATB: Antibiótico. 
fección del tracto urinario en ambos grupos (cesárea electiva y de urgencia) (9). En $\mathrm{HCUCH}$, entre 93 y $98 \%$ de las pacientes sometidas a cesárea recibieron profilaxis antibiótica, sin embargo sólo en $83 \%$ de las cesáreas electivas y $72 \%$ de las de urgencia se indicó cefazolina 2 gramos por una vez.

En el caso de parto vaginal, las recomendaciones internacionales no indican el uso de antibióticos, excepto cuando se trata de parto vaginal operatorio. Hay estudios que describen un aumento en la incidencia de infecciones postparto y readmisión materna al Hospital en partos operatorios, por lo que se justificaría el uso de profilaxis antibiótica. Se comparó pacientes que recibieron antibiótico v/s un grupo control y se observó que no hubo diferencias estadísticamente significativas en la reducción de infecciones y readmisión materna, pero si hubo una disminución del riesgo relativo en $93 \%$. Sin embargo, la calidad del estudio es insuficiente como para hacer una recomendación (10). En el HCUCH, sólo $60 \%$ de las pacientes con parto operatorio recibió antibióticos, cifra concordante con la falta de estudios que recomiendan su uso. Es importante destacar que en la mayoría de los partos vaginales espontáneos se indico profilaxis antibiótica, a diferencia de lo descrito en la literatura. Esto se puede entender, porque en casi la totalidad de los partos se realiza una revisión instrumental de la cavidad uterina, es decir, un legrado uterino, por lo que habría indicación de antibióticos.

Finalmente, al analizar el uso de antibióticos en vaciamientos uterinos por abortos espontáneos o sospecha de abortos inducidos, se ha descrito el uso de profilaxis para evitar complicaciones como procesos inflamatorios pelvianos o infertilidad secundaria. Un estudio evaluó la efectividad del uso de antibióticos en abortos incompletos y determinó que no hay evidencia suficiente que avale su uso por falta de estudios con buen seguimiento de pacientes (11). En $\mathrm{HCUCH}$, solo 63 y $75 \%$ de los vaciamientos de primer y segundo trimestre respectivamente, recibieron profilaxis antibiótica. Al comparar el tipo de antibiótico recomendado con el esquema indicado en nuestro hospital, se observó que en vaciamientos del pri- mer trimestre se utilizó mayoritariamente cefazolina y en ningún caso doxiciclina como se describe en normas internacionales. Sólo tuvimos 8 casos de vaciamientos de segundo trimestre, en los cuales se usó principalmente cefazolina 2 gramos, siendo la recomendación del NIH 1 gramo.

Podemos concluir que hay estudios que recomiendan el uso de profilaxis antibiótica en todas las cesáreas electivas y de urgencia, sin embargo, aún no existe consenso en otros procedimientos como en parto operatorio o vaciamientos uterinos. Son necesarios más estudios randomizados y controlados para poder normar el uso de antibióticos profilácticos en estos procedimientos.

\section{BIBLIOGRAFÍA}

1. Weed H. Antimicrobial prophylaxis in the surgical patient. Med Clin N Am 2003; 87: 59-75.

2. Antimicrobial prophylaxis in surgery. Med Lett Drugs Ther 2001; 43: 92-7.

3. Chelmow D, Ruehli M, Huang E. Prophylactic use of antibiotics for non laboring patients undergoing cesarean delivery with intact membranes: a metaanalysis. Am J Obstet Gynecol 2001; 184: 656-61.

4. Sawaya G, Grady D, Kerlikowske K, et al. Antibiotics at the time of induced abortion: the case for universal prophylaxis based on a meta-analysis. Obstet Gynecol 1996; 87: 884-90.

5. Control de Infecciones Intrahospitalarias, Productos Farmacéuticos y Normas Transfusionales. Hospital Clínico de la Universidad de Chile 2004-2007.

6. National Institute of Health. Profilaxis Antibiótica. Hallado en: http://www.nih.org

7. Anuario 2004. División de Operaciones. Hospital Clínico de la Universidad de Chile.

8. Hopkins L, Smaill F. Antibiotic prophylaxis regimens and drugs for cesarean section. Cochrane Database Systematic Reviews, 1, 2005.

9. Smaill F, Hofmeyr GJ. Antibiotic prophylaxis for cesarean section. Cochrane Database Systematic Reviews, 1, 2005.

10. Liabsuetrakul T, Choobun T, Peeyananjarassri K, Islam M. Antibiotic prophylaxis for operative vaginal delivery. Cochrane Database Systematic Reviews, 1, 2005.

11. May W, Gulmezoglu AM, Ba-Thike K. Antibiotics for incomplete abortion. Cochrane Database Systematic Reviews, 1, 2005 